Pathophysiology

of Haemostasis and Thrombosis
Pathophysiol Haemost Thromb 2007-08;36:282-284

DOI: $10.1159 / 000252826$

\title{
Thrombophilia and Transient Risk Factors for Venous Thromboembolism in Women with Distal Deep Vein Thrombosis
}

\author{
S. Cozza ${ }^{a} \quad$ G. Mercier ${ }^{b} \quad$ J.F. Schved ${ }^{a} \quad$ C. Biron-Andréani ${ }^{a}$ \\ ${ }^{a}$ Laboratoire d'Hématologie and ${ }^{b}$ Département d'Informatique Médicale, CHRU Montpellier, Montpellier, France
}

Dear Sir,

Although deep vein thrombosis (DVT) and its major complication, pulmonary embolism (PE), are usually considered a single disease entity, venous thromboembolism (VTE) has a wide spectrum of clinical manifestations. A variety of risk factors may play different roles in determining the various locations of thrombi [1]. For example, the factor II 20210A (FII20210A) polymorphism has been reported to be more than twice as common in patients with cerebral venous sinus thrombosis than in patients with DVT [2]. Several studies have provided evidence for a reduced incidence of PE in relation to DVT in patients with the factor V Leiden (FVL) mutation [3-5]. Recently, 3 studies accessing the rate of thrombophilia and the location of lower-extremity DVT reported discordant results [6-8]. Patients with a deficiency in antithrombin (AT), protein $\mathrm{C}(\mathrm{PC})$ or protein $\mathrm{S}(\mathrm{PS})$, or a high level of factor VIII (FVIII) were excluded from these studies. In the present study, we investigated whether thrombophilic defects including AT, PC, PS deficiency or a high FVIII level play a different role in patients presenting with isolated PE, distal or proximal DVT. We also analyzed the association between the presence of transient risk factors at the time of VTE and the location of VTE.
We retrospectively investigated consecutive women aged 12-49 years diagnosed with VTE and referred to our Thrombosis Center to be investigated for thrombophilia screening in the last 3 years. DVT was objectively confirmed by bilateral B-mode compression ultrasound and $\mathrm{PE}$ by ventilation/perfusion lung scan, computed tomography or angiography. Patients underwent objective investigation for PE only if they had pulmonary symptoms, whereas patients with symptomatic PE underwent objective investigation for DVT. DVT of the calf vein was considered distal, thrombosis involving the remaining vein segments was considered proximal. The transient risk factors considered were: surgery, trauma, leg cast, oral contraceptives, hormonal replacement therapy, pregnancy and puerperium. We previously described the methods used for thrombophilia screening [9]. All subjects gave their informed consent to participate in the study in accordance with the Local Ethics Committee Recommendations.

We analyzed the presence of consensual thrombophilia and transient risk factors in 47 women with distal DVT complicated $(n=6)$ by PE or not $(n=41), 47$ with proximal DVT complicated $(\mathrm{n}=5)$ by symptomatic PE or not $(n=42)$, and 21 women with isolated PE. Women with isolated PE were younger at the time of the first event (mean age 24.5 years) than those with

Christine Biron-Andréan

Laboratoire d'Hématologie, Hôpital Saint-Eloi, CHRU Montpellier 80, avenue Augustin Fliche

FR-34295 Montpellier Cedex 5 (France)

Tel. +33467337 031, Fax +33467337 036, E-Mail c-biron@chu-montpellier.fr
2009 S. Karger AG, Basel

$1424-8832 / 08 / 0365-0282 \$ 24.50 / 0$

www.karger.com/pht 
Table 1. Frequency of the different types of thrombophilia according to the location of venous thromboembolism

\begin{tabular}{llcc}
\hline & $\begin{array}{l}\text { Isolated PE } \\
(\mathrm{n}=21)\end{array}$ & $\begin{array}{l}\text { Distal DVT } \\
(\mathrm{n}=47)\end{array}$ & $\begin{array}{l}\text { Proximal DVT } \\
(\mathrm{n}=47)\end{array}$ \\
\hline Thrombophilic defect $^{\text {Natural anticoagulant deficiency }}{ }^{1}$ & $9(42.9)$ & $14(29.8)$ & $18(38.3)$ \\
Factor V Leiden & $4(19)$ & $2(4.3)$ & $4(8.5)$ \\
FII20210A polymorphism & $2 \pm 9.5$ & $3 \pm 6.4$ & $3 \pm 6.4$ \\
Elevated FVIII >200 U/dl $_{\text {Antiphospholipid antibodies }}$ & $3 \pm 14.3$ & $4 \pm 8.5$ & $6 \pm 12.8$ \\
Combined thrombophilia $^{2}$ & 0 & $3 \pm 6.4$ & $1 \pm 2.13$ \\
\hline
\end{tabular}

\footnotetext{
Figures in parentheses are percentages.

${ }^{1}$ Natural anticoagulant deficiencies include AT, PC or PS deficiency; none of the patients with deficiency had a type 1 AT defect.

${ }^{2}$ Combined thrombophilia included heterozygous FVL and FII20210A, FVL and high-level FVIII, FVL and heparin binding site AT deficiency.
}

distal DVT (30.7 years) or proximal DVT (29.4 years; $\mathrm{p}=0.008)$.

No significant difference in the presence or absence of transient risk factors present at the time of VTE was observed in the 3 groups of patients $(p=0.5)$; oral contraceptive use was reported in $67 \%$ of the distal events, $49 \%$ of the proximal events or $57 \%$ of isolated PE ( $\mathrm{p}=0.15)$.

Thrombophilia results were abnormal in $35.7 \%$ (41/115), including lupus anticoagulant/anticardiolipid antibodies ( $\mathrm{n}=2,1.7 \%)$, AT, PC or PS deficiency $(\mathrm{n}=10$, 8.7\%), heterozygous FVL mutation ( $\mathrm{n}=8,7 \%)$, heterozygous FII20210A polymorphism $(\mathrm{n}=13,11.3 \%)$, high FVIII level ( $\mathrm{n}=4,3.5 \%)$, and combined thrombophilia $(\mathrm{n}=4,3.5 \%)$. The proportion of any of the types of thrombophilia tested between women with distal DVT, proximal DVT or isolated PE was statistically not different $(\mathrm{p}=0.68$; table 1$)$.

In the present study, we compared the role of the different thrombophilic defects and the presence of transient risk factors in 3 groups of women with distal DVT, proximal DVT, whether or not complicated by PE, and isolated PE. As previously reported by Martinelli et al. [7], the presence of transient risk factors was similar in the 3 groups. Margaglione et al. [5] found that the presence of circumstantial risk factors was significantly higher in patients with isolated PE compared with those with DVT with PE, but he did not compare distal and proximal DVT. Concerning thrombophilia, we did not find any statistically significant difference. To our knowledge, our report is the first study that has investigated an elevated FVIII level and location of VTE.

Risk Factors for Thromboembolism in Women with Distal DVT
As for natural anticoagulant deficiencies, we found only an Italian series where these defects were observed in $4.9 \%$ in a DVT group and in $0.8 \%$ in an isolated PE group $(\mathrm{p}=0.026)$ [5]; distal and proximal DVTs were not compared. The differential anatomic distribution has been mainly described for the FVL mutation. Although several studies have reported a reduced incidence of $\mathrm{PE}$ in patients with the FVL mutation, in a large Danish cohort study, a reduced risk associated with this type of thrombophilia was not demonstrated [10]. Moreover, the explanation for the weak association between FVL mutation and isolated PE remains unclear [1].

Comparing distal and proximal DVTs, there is inconclusive evidence from 3 recently published studies [6-8]. In proximal DVTs, we found the same frequency of FVL mutation as previously reported by Huisman et al. [6] and Schulman [8]. In isolated calf vein thrombosis, Schulman [8] and Martinelli et al. [7] reported similar levels of frequency, while Huisman et al. [6] found prevalence of the FVL mutation to be twice as high, at $11.8 \%$. The FII20210A polymorphism has not been fully described, and the data are controversial $[5,7]$. Our results show that FII20210A was more frequent in isolated PE (14.3\%) and proximal DVT (12.8\%) than in distal DVT (8.5\%), but the difference did not reach statistical significance. It cannot be ruled out that differences in diagnostic means and selection bias could explain the discrepancies between the studies. In terms of age at occurrence of the first VTE, isolated PE appears to be associated with a more severe clinical profile. 
Our study has some limitations: the highly selected young population referred for a thrombophilia screening, the small number of patients, the retrospective design and the diagnostic techniques (PE was searched for only if symptoms were present; the accuracy of compression ultrasound was lower in distal than in proximal DVTs).
In conclusion, the results of this study suggest that the frequency of the different thrombophilic defects is not significantly different in patients with distal DVT, proximal DVT, whether or not complicated by PE, or isolated PE. Due to the limitations of the study, the data should be interpreted with caution.

\section{References}

1 Perrier A: Deep vein thrombosis and pulmonary embolism: a single disease entity with different risk factors? Chest 2000;118:12341236.

2 Wysokinska EM, Wysokinski WE, Brown RD, Karnicki K, Gosk-Beirska I, Grill D, McBane RD 2nd: Thrombophilia differences in cerebral venous sinus and lower extremity deep venous thrombosis. Neurology 2008; 70:627-633.

3 de Moerloose P, Reber G, Perrier A, Perneger $\mathrm{T}$, Bounameaux H: Prevalence of factor $\mathrm{V}$ Leiden and prothrombin G20210A mutations in unselected patients with venous thromboembolism. Br J Haematol 2000;110: 125-129.
4 Lindmarker P, Schulman S, Sten-Linder M, Wiman B, Egberg N, Johnsson H: The risk of recurrent venous thromboembolism in carriers and non-carriers of the G1691A allele in the coagulation factor $\mathrm{V}$ gene and the G20210A allele in the prothrombin gene. DURAC Trial Study Group. Duration of Anticoagulation. Thromb Haemost 1999;81: 684-689.

5 Margaglione M, Brancaccio V, De Lucia D, Martinelli I, Ciampa A, Grandone E, Di Minno G: Inherited thrombophilic risk factors and venous thromboembolism: distinct role in peripheral deep venous thrombosis and pulmonary embolism. Chest 2000;118: 1405-1411.

6 Huisman MV, Klok FA, Karami Djurabi R, Tormene D, Simioni P, Prandoni P: Factor V Leiden is associated with more distal location of deep vein thrombosis of the leg. J Thromb Haemost 2008;6:544-545.
7 Martinelli I, Battaglioli T, Razzari C, Mannucci PM: Type and location of venous thromboembolism in patients with factor $\mathrm{V}$ Leiden or prothrombin G20210A and in those with no thrombophilia. J Thromb Haemost 2007;5:98-101.

8 Schulman S: Thrombophilia and location of venous thromboembolism. J Thromb Haemost 2007;5:2151-2152.

9 Procare Group: Is recurrent venous thromboembolism more frequent in homozygous patients for the factor $\mathrm{V}$ Leiden mutation than in heterozygous patients? Blood Coagul Fibrinolysis 2003;14:523-529.

10 Juul K, Tybjaerg-Hansen A, Schnohr P, Nordestgaard BG: Factor V Leiden and the risk for venous thromboembolism in the adult Danish population. Ann Intern Med 2004; 140:330-337. 